\title{
The study of blood transcriptome profiles in Holstein cows with miscarriage during peri-implantation
}

\author{
Guoli Zhao', Yanyan Li ${ }^{2}$ Xiaolong Kang ${ }^{1}$, Liang Huang ${ }^{2}$, Peng $\mathrm{Li}^{1}$, Jinghang Zhou', and Yuangang Shi',*
}

* Corresponding Author: Yuangang Shi Tel: +86-9512061874, Fax: +86-9512061874,

E-mail: shyga818@126.com

${ }^{1}$ Department of Animal Husbandry, Agricultural College of Ningxia University, Yinchuan, Ningxia 75004, China

2 Helan Mountain Diary Company of Ningxia, Yinchuan, Ningxia 75004, China

ORCID

Yuangang Shi

https://orcid.org/0000-0002-9161-3392

Submitted Oct 24, 2017; Revised Mar 15, 2018; Accepted May 24, 2018
Objective: In this study, the transcriptome profile of cow experiencing miscarriage during peri-implantation was investigated.

Methods: Total transcriptomes were checked by RNA sequencing, and the analyzed by bioinformatics methods, the differentially expressed genes (DEGs) were analysed with hierarchical clustering and Kyoto encyclopedia of genes and genomes (KEGG) pathway analysis.

Results: The results suggested that serum progesterone levels were significantly decreased in cows that miscarried as compared to the pregnant cows at 18,21,33,39, and 51 days after artificial insemination. The RNA sequencing results suggested that 32, 176, 5, 10, and 2 DEGs were identified in the pregnant cows and miscarried cows at $18,21,33,39$, and $51 \mathrm{~d}$ after artificial insemination. And 15, 101, 1,2, and 2 DEGs were upregulated, and 17, 74, 4, and 8 DEGs were downregulated in the cows in the pregnant and miscarriage groups, respectively at 18,21 , 33 , and 39, but no gene was downregulated at $51 \mathrm{~d}$ after artificial insemination. These DEGs were distributed to $13,20,3,6$, and 20 pathways, and some pathway essential for pregnancy, such as cell adhesion molecules, tumor necrosis factor signaling pathway and PI3K-Akt signaling pathway.

Conclusion: This analysis has identified several genes and related pathways crucial for pregnancy and miscarriage in cows, as well as these genes supply molecular markers to predict the miscarriage in cows.

Keywords: Differentially Expressed Genes; Miscarriage; Pregnancy; Progesterone; RNA Sequencing

\section{INTRODUCTION}

For a successful pregnancy, the blastocyst's implantation to the maternal endometrium is crucial [1]. The rate of miscarriage caused by embryonic and fetal losses is approximately $40 \%$ in cattle [2-4]. Previous study suggested that many genes and signalling pathway are involved in early pregnancy, and some genes are essential for maintaining pregnancy, and the miscarriage was triggered by the dysfunction of these genes, such as cadherin $2(\mathrm{CDH} 2)$, which is a gene of cell adhesion molecules (CAMs) [5]; collagen type VI alpha 3 chain (COL6A3), thrombospondin 1 (THBS1), fibronectin 1, integrin beta 4 (ITGB4) [6], which are all PI3KAkt signaling pathway genes; matrix metallopeptidase 14 (MMP14), vascular cell adhesion molecule 1 (VCAM1) [7], matrix metallopeptidase 9 (MMP9), which are all tumor necrosis factor (TNF) signaling pathway genes; additionally, MAPK signaling pathway [8] and Hippo signaling pathway [9] are involved in maintaining pregnancy. In addition, miscarriage was caused by infectious diseases such as bovine herpesvirus 1 and brucellosis [10,11]. The miscarriage event eventually induces damages in the pregnancy mechanism of the cow. For this reason, studying the prevention and diagnosis of bovine miscarriages greatly attracted researchers in the past. 
The steroid hormone progesterone $\left(\mathrm{P}_{4}\right)$ is essential for the establishment and maintenance of pregnancy [12]. Elevated concentrations of $\mathrm{P}_{4}$ during the post-conception period generally result in higher pregnancy rate and greater conceptus survival $[13,14]$. Therefore, the concentration of $\mathrm{P}_{4}$ can be used as a marker for determining the early stages of pregnancy.

Elevated concentrations of circulating $\mathrm{P}_{4}$ have been associated with an advancement of conceptus elongation, and an elongated conceptus is immediately implanted to endometrial epithelial cells in cattle between 18 and $19 \mathrm{~d}$ [15]. Therefore, the endometrium and the embryos of pregnant cows at 18 to $19 \mathrm{~d}$ were used to detect the related genes involved in the pregnancy. However, it is difficult to collect endometrium and embryo samples in cows, which adds to the difficulty in diagnosing miscarriage and pregnancy. Blood serum can provide an optimal sample to explore genes involved in pregnancy and miscarriage. Thus, we aimed to study the transcriptome of blood serum samples of both miscarried and pregnant cows, through RNA sequencing, which could help us better understand any novel marker genes responsible for diagnosing pregnancy and miscarriage.

\section{MATERIALS AND METHODS}

\section{Selection of cows}

Primiparous holstein cows aged 12 to 18 months were selected for this study. These cows without history of reproductive ailments or infectious diseases, were naturally coming in heat and brought for artificial insemination (AI). All animals were provided with balanced ration and ad libitum water throughout the day. Physiological responses were examined regularly to ensure healthy status of the cows. Animal pregnancy was ensured by checking of nonreturn of heat and progesterone level (through ELISA test), whereas miscarriage was confirmed through the examination of embryonic and endometrium dysfunctions/symptoms. All procedures regarding animal care and treatments were approved by the Committee for Experimental Animals of Ningxia University.

\section{Collection of blood}

The blood of pregnant and miscarriaged cows at $18 \mathrm{~d}, 21 \mathrm{st}$, $33 \mathrm{rd}, 39 \mathrm{~d}$ and 51 st post-AI was collected according to previous study [16], at least 6 cows were used to collect blood in each group. Each of the blood samples, after collection, was centrifuged for $10 \mathrm{~min}$ at 3,000 rpm and separated into 3 layers. The blood serum and leukocytes residing in the upper and middle layers, respectively, were collected gently by RNase-free tips. For leukocytes, an additional Trizol reagent (Takara, Dalian, China) was also added with it in a 1.5-mL RNase-free centrifuge tube. The blood serum, leukocytes were preserved as liquid for progesterone estimation and RNA sequencing, and polymerase chain reaction (PCR).
RNA extraction, RNA sequencing, real-time PCR and ELISA

Total RNA was extracted using Trizol reagent according to the manufacturer's instructions. The quality of RNA was observed with a nano-drop 2000 spectrophotometer (RNA concentration and purity) and DNA agarose gel (RNA integrity). Finally, those of good RNA samples were used for sequencing and real-time PCR.

The RNA sequencing was performed by Novogene Company (Beijing, China) following standard protocols.

For PCR analysis, the DNA was reverse-transcribed using PrimeScript RT reagent kit (Takara, China) according to the manufacturer's instructions. The real-time PCR procedure was performed with ABI7500 Fast, and the data were analysed by ABI7500 Fast software. A $10 \mu \mathrm{L}$ SYBR Premix Ex Taq II was used for detection of PCR product from a $20 \mu \mathrm{L}$ volume mixture that also included $2.0 \mu \mathrm{L}$ complementary DNA ( $\approx 25 \mathrm{ng}$ total RNA), $0.8 \mu \mathrm{L}$ of each primer at $10 \mathrm{mM}$, $0.4 \mu \mathrm{L}$ ROX reference dye, and $6 \mu \mathrm{L}$ of nuclease-free water. The PCR run included a 1 cycle run at $95^{\circ} \mathrm{C}$ for $30 \mathrm{~s}$, a $40 \mathrm{cy}$ cles run at $95^{\circ} \mathrm{C}$ for $5 \mathrm{~s}$, which followed by another 40 cycles run at $60^{\circ} \mathrm{C}$ for $30 \mathrm{~s}$.

Gene mRNA quantification was performed by the $2^{-\Delta \Delta \mathrm{Ct}}$ method according to previous study [17], and glyceraldehyde3-phosphate dehydrogenase was used as a reference gene to normalize the target gene expression. The primer of genes was shown in Table 1.

The blood serum concentration of progesterone was measured by an ELISA kit following manufacturer's protocol at the Beijing Sino-uk Institute of Biological Technology with an ELISA kit according to the manufacturer's instructions.

\section{Statistical analysis}

Experiments on each group of animals were replicated ( 3 times or more) for more accurate estimation. Data obtained in the study were analyzed with GraphPad Prism 5 using paired $t$ tests. Significant difference was defined as $\mathrm{p}<0.05$.

\section{RESULTS}

\section{Progesterone levels}

Figure 1(a) to 1(e) showed that the progesterone concentration was significantly lower in cows who miscarried than in pregnant cows at $18 \mathrm{~d}$ post-AI (Figure 1a; $\mathrm{p}<0.01$ ), at $21 \mathrm{~d}$ postAI (Figure 1b; $p<0.05$ ), at $33 \mathrm{~d}$ (Figure $1 \mathrm{c} ; \mathrm{p}<0.001$ ), at $39 \mathrm{~d}$ (Figure 1d; $\mathrm{p}<0.001$ ) and at $51 \mathrm{~d}$ (Figure 1e; $\mathrm{p}<0.001$ ). In addition, the pregnancy and miscarriage were further confirmed by B-ultrasonic diagnosis (data not shown).

\section{Differentially expressed genes in cows}

RNA sequencing in cows on 18th post-AI: Hierarchical clustering of differentially expressed genes (DEGs) showed that DEG 
Table 1. Primers for real time polymerase chain reaction

\begin{tabular}{|c|c|}
\hline Gene name & Primers \\
\hline \multirow[t]{2}{*}{ LILRA4 } & Forward: GCCAGGTTCTCCATCCAGTA \\
\hline & Reverse: CTCCTTCCTGTCACCACCAG \\
\hline \multirow[t]{2}{*}{$\mathrm{CDH} 2$} & Forward: CCTCTGGATCGTGAGCTGAT \\
\hline & Reverse: CCCTCAGGAACTGTCCCATT \\
\hline \multirow[t]{2}{*}{ MMP14 } & Forward: GCCTTGAGCATTCCAACGAT \\
\hline & Reverse: GCACGAAGTTCTCTGTGTCC \\
\hline \multirow[t]{2}{*}{ VCAM1 } & Forward: TACCAGCTCCACGGATTCTC \\
\hline & Reverse: CCCAGAATCTTCTGCCCTCA \\
\hline \multirow[t]{2}{*}{ LOC100139209 } & Forward: TCGTCACCTCTGGACAGAAC \\
\hline & Reverse: TTGGACAGAGCGAATCTGGT \\
\hline \multirow[t]{2}{*}{ EEF1A1 } & Forward: AACTCGCCCAACTGACAAAC \\
\hline & Reverse: CCCACAGGGACAGTACCAAT \\
\hline \multirow[t]{2}{*}{ PRSS2 } & Forward: CTCCAGGGCATTGTGTCTTG \\
\hline & Reverse: TCCACGTAGTTGCAGACCTT \\
\hline \multirow[t]{2}{*}{ BoLA-DQA } & Forward: AGGTTCCAGAGGTGACTGTG \\
\hline & Reverse: ACGTGACAGATGAGGGTGTT \\
\hline \multirow[t]{2}{*}{$B O L A-D Q B$} & Forward: TGCTCGGTGACGGATTTCTA \\
\hline & Reverse: CTCCAGCATCACGAGGATCT \\
\hline \multirow[t]{2}{*}{ GAPDH } & Forward: CATGACCACTTTGGCATCGT \\
\hline & Reverse: CCATCCACAGTCTTCTGGGT \\
\hline
\end{tabular}

LILRA4, leukocyte immunoglobulin like receptor $\mathrm{A4}$; $\mathrm{CDH2}$, cadherin 2; MMP14, matrix metallopeptidase 14; VCAM1, vascular cell adhesion molecule 1; LOC100139209, leukocyte immunoglobulin-like receptor subfamily A member 6; EEF1A1, eukaryotic translation elongation factor 1 alpha 1; PRSS2, serine protease 2; BoLA-DQA, BOS taurus MHC class II antigen $\mathrm{A}$; GAPDH, glyceraldehyde-3-phosphate dehydrogenase.

expression patterns on 18th were different between the pregnant and miscarriage cows (Figure 2a). DEGs were screened in a volcano plot, and results suggested that 32 DEGs were found; 15 were upregulated and 17 were downregulated (Figure $2 b$ ). Kyoto encyclopedia of genes and genomes (KEGG) pathway analysis showed that DEGs were distributed in 13 pathways (Figure 2c). In addition, the expression of two DEGs, leukocyte immunoglobulin like receptor A4 (LILRA4) which belongs to natural killer cell mediated cytotoxicity pathway, and $\mathrm{CDH} 2$ belongs to cell adhesion molecules pathway, two genes are all closely correlated with pregnancy, was confirmed by real-time PCR, a finding consistent with the RNA sequencing results. These two genes were significantly downregulated in the miscarriage cow group than in the pregnant cow group (Figure 7a, b; $\mathrm{p}<0.05$ ).

$R N A-$ sequencing in cows on 21st post-AI: Hierarchical clustering of DEGs showed that DEG expression patterns in $21 \mathrm{~d}$ were different between the pregnant and miscarriage cow groups (Figure 3a). DEGs were screened in a volcano plot, and results suggested that 175 DEGs were found; 101 were upregulated and 74 were downregulated (Figure 3b). KEGG pathway analysis showed that DEGs were distributed in 20 pathways (Figure 3c). The expression of two DEGs, MMP14 and VCAM1 belong to TNF signaling pathway, which are closely correlated with pregnancy, was confirmed by real-time PCR, a finding consistent with the RNA sequencing results, the two genes closely correlated with pregnancy, MMP14 and VCAM1, were significantly upregulated in the miscarriage cow group than in the pregnant cow group (Figure $7 \mathrm{c}, \mathrm{d} ; \mathrm{p}<0.05$ ).

RNA sequencing in cows on 33rd, 39th, and 51st post-AI: Compared with results of hierarchical clustering of DEGs at 18 and $21 \mathrm{~d}$, hierarchical clustering of DEGs showed that DEG expression patterns at $33 \mathrm{~d}$ (Figure 4a), $39 \mathrm{~d}$ (Figure 5a), and $51 \mathrm{~d}$ (Figure 6a) were weakly different between the pregnant and miscarriage cow groups. Volcano plot results suggested that 5 DEGs were found at $33 \mathrm{~d} ; 1$ was upregulated and 4 were downregulated (Figure 4b), KEGG pathway analysis showed that DEGs were distributed in 3 pathways (Figure 4c); 10 DEGs were found at $39 \mathrm{~d}, 2$ were upregulated, and 8 were downregulated (Figure $5 b$ ). KEGG pathway analysis showed that DEGs were distributed in 6 pathways (Figure 5c); 2 DEGs were found at $51 \mathrm{~d}, 2$ were upregulated, and no DEG was downregulated (Figure 6b). KEGG pathway analysis showed that DEGs were involved in 20 pathways (Figure 6c). To further confirm the results of RNA sequencing, two genes, namely LOC100139209 (leukocyte immunoglobulin-like receptor subfamily A member 6) and LILRA4 at $33 \mathrm{~d}$ (Figure 7e, 7f), eukaryotic translation elongation factor 1 alpha 1 and PRSS2 (serine protease 2) at $39 \mathrm{~d}$ (Figure 7g, 7h), and Bos taurus $\mathrm{MHC}$ class II antigen A (BoLA-DQA) and BoLA-DQB (Figure 7i, 7j) were checked by real-time PCR, and results suggested that only the expression of BoLA-DQB was not consistent with the results of RNA sequencing.

\section{DISCUSSION}

Approximately $50 \%$ of miscarriages occur during the process of embryo implantation, when blastocyst hatching and embryo attachment occur in the endometrium of cattle [18]; the establishment of proper maternal-fetal communication and optimal uterine environment are essential. Although various studies were implemented to explore the expression of transcripts and proteins in bovine endometria and conceptus [1921], the mechanism of pregnancy and miscarriage was not fully elucidated.

Our results revealed that the progesterone concentration in cows who miscarried was remarkably decreased compared with that during pregnancy. In mammals, including cows, the continued $\mathrm{P}_{4}$ secretion is indispensable for the establishment and maintenance of pregnancy [22]. In this regard, a report by Psychoyos [23], stated that various genes expressions that were directly or indirectly regulated by $\mathrm{P} 4$ in endometrium were involved with the regulation of numerous uterine functions for normal embryonic development through endometrial secretions. Therefore, elevated progesterone concentration maintains the pregnancy, and the miscarriage event in the cow was triggered by decreased progesterone level.

Our studied serum transcriptome profiles in cows provided 

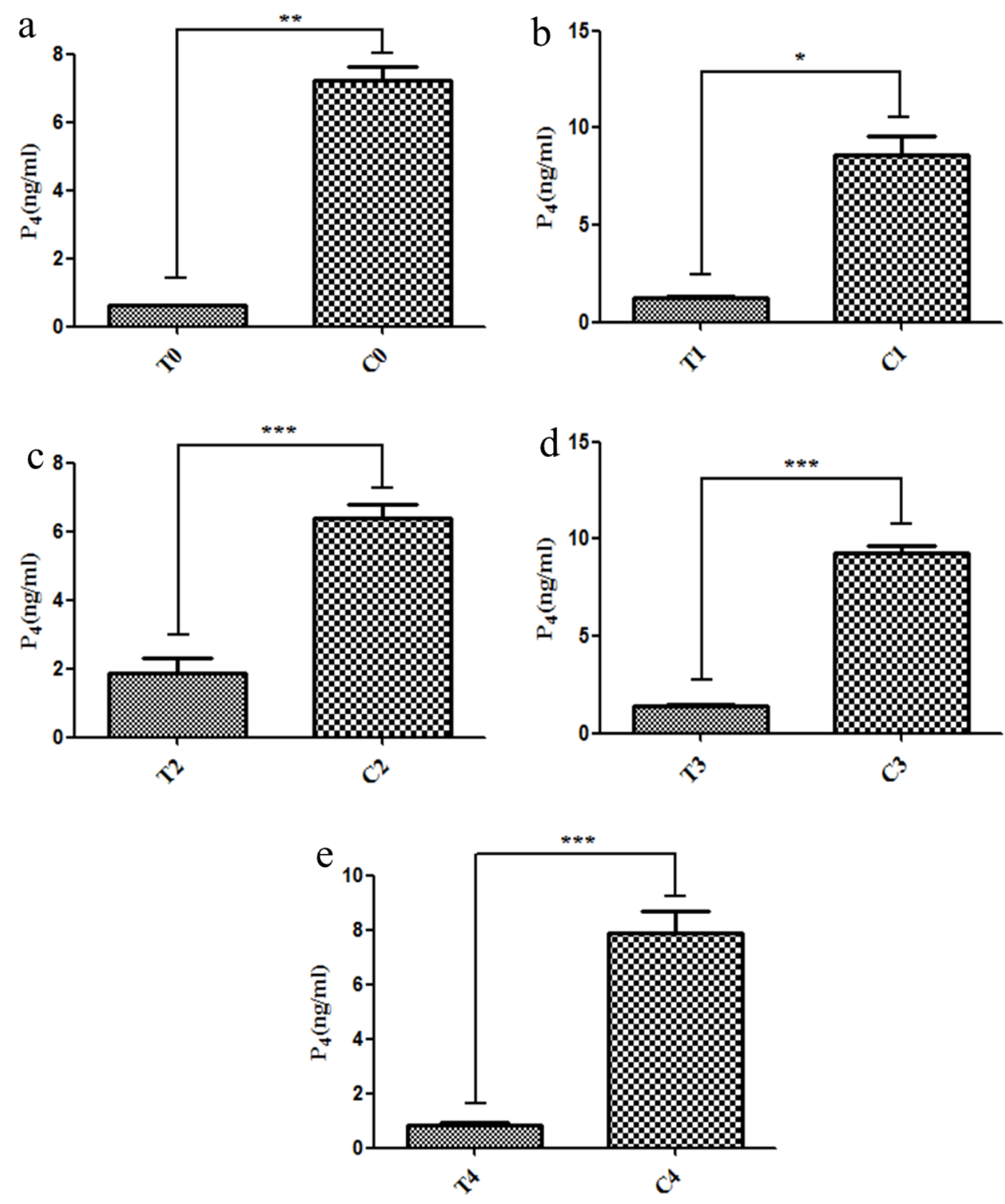

Figure 1. The progesterone levels in pregnant cows and cows who miscarried. The progesterone $\left(\mathrm{P}_{4}\right)$ levels were significantly decreased in the miscarriage cow group than in the pregnant cow group at $18 \mathrm{~d}(\mathrm{a}), 21 \mathrm{~d}(\mathrm{~b}), 33 \mathrm{~d}(\mathrm{c}), 39 \mathrm{~d}(\mathrm{~d})$, and $51 \mathrm{~d}(\mathrm{e})$. T, miscarriage cow group; C, pregnant cow group. ${ }^{*} p<0.05,{ }^{* *} p<0.01,{ }^{* * *} p<0.001$.

a greater insight into the function of various DEGs at different pregnancy periods, and results suggested that several DEGs were identified in pregnant cows and miscarried cows at 18 and $21 \mathrm{~d}$, but only a few DEGs were identified at 33, 39, and $51 \mathrm{~d}$, and this might be attributed to the embryo implantation occurred on the 18 to $19 \mathrm{~d}$ post-AI [15], and 50\% of miscarriages in cows occur during the process of embryo implantation [18]; indeed, the embryo implantation was regulated by many embryonic and endometrium genes. At $18 \mathrm{~d}, 32$ DEGs involved in pregnancy and miscarriage were identified, such as LILRA4, LILRA6, leukocyte immunoglobulin-like receptor subfamily
B member 3, leukocyte immunoglobulin-like receptor subfamily A member 6, DNA-directed RNA polymerase I subunit RPA12, CDH2, natural cytotoxicity triggering receptor 1 , Bos taurus 2',5'-oligoadenylate synthetase 1 (OAS1Y, 40/46kDa), and carbonic anhydrase 5A; in addition, the importance of $\mathrm{CDH} 2$ on embryo implantation was emphasized by previous study [24]. Furthermore, 175 DEGs were identified at $21 \mathrm{~d}$, and those genes are mainly distributed in 20 pathways, such as PI3K-Akt signalling pathway, tumour necrosis factor signalling pathway, MAPK signalling pathway, and Hippo signalling pathway, COL6A3, THBS1, fibronectin 1, and ITGB4 are all 
Cluster analysis of genes
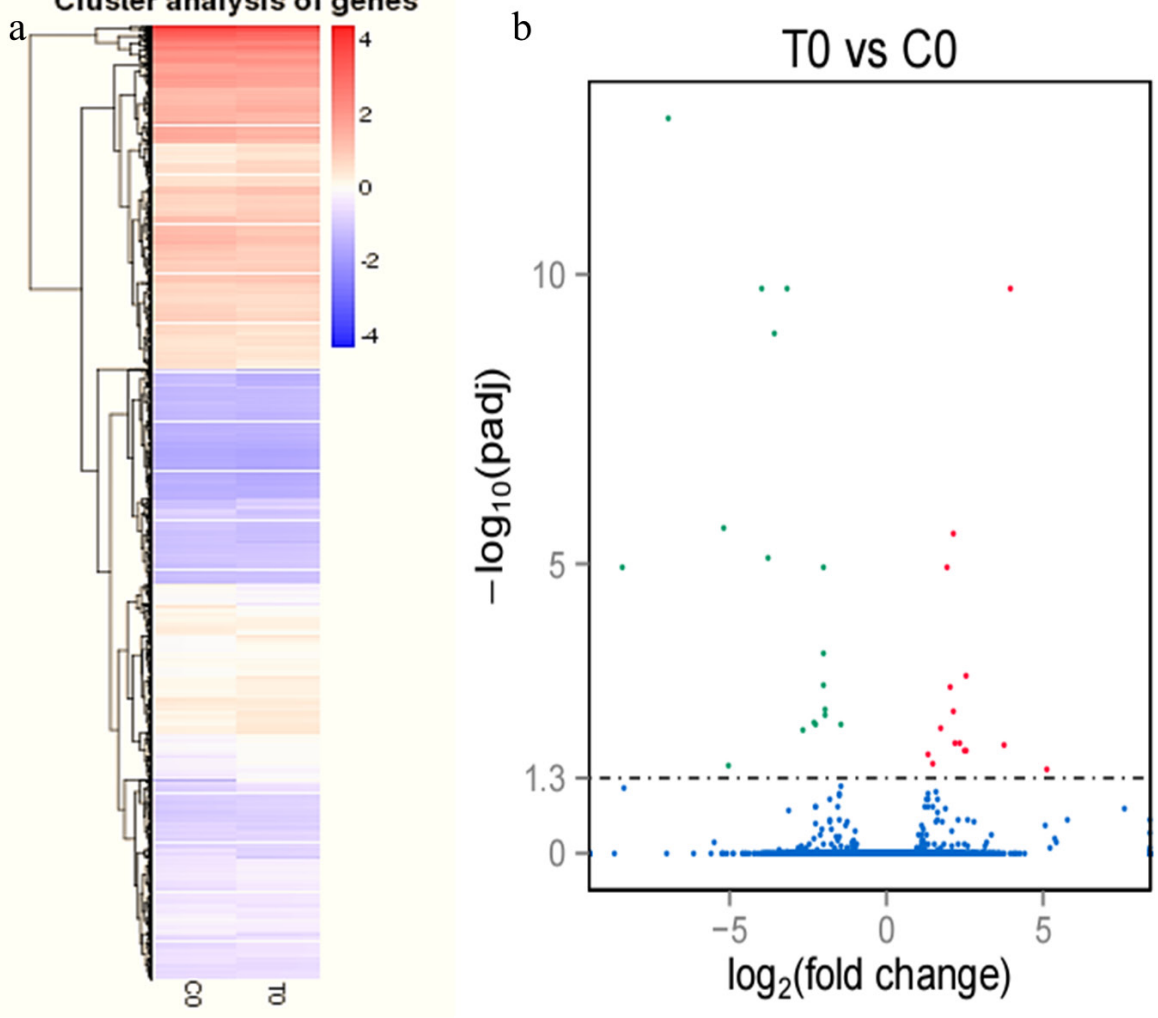

\section{Differential Expressed Genes ( 32 )}

- up regulated: 15

- down regulated: 17

$\mathrm{c}$
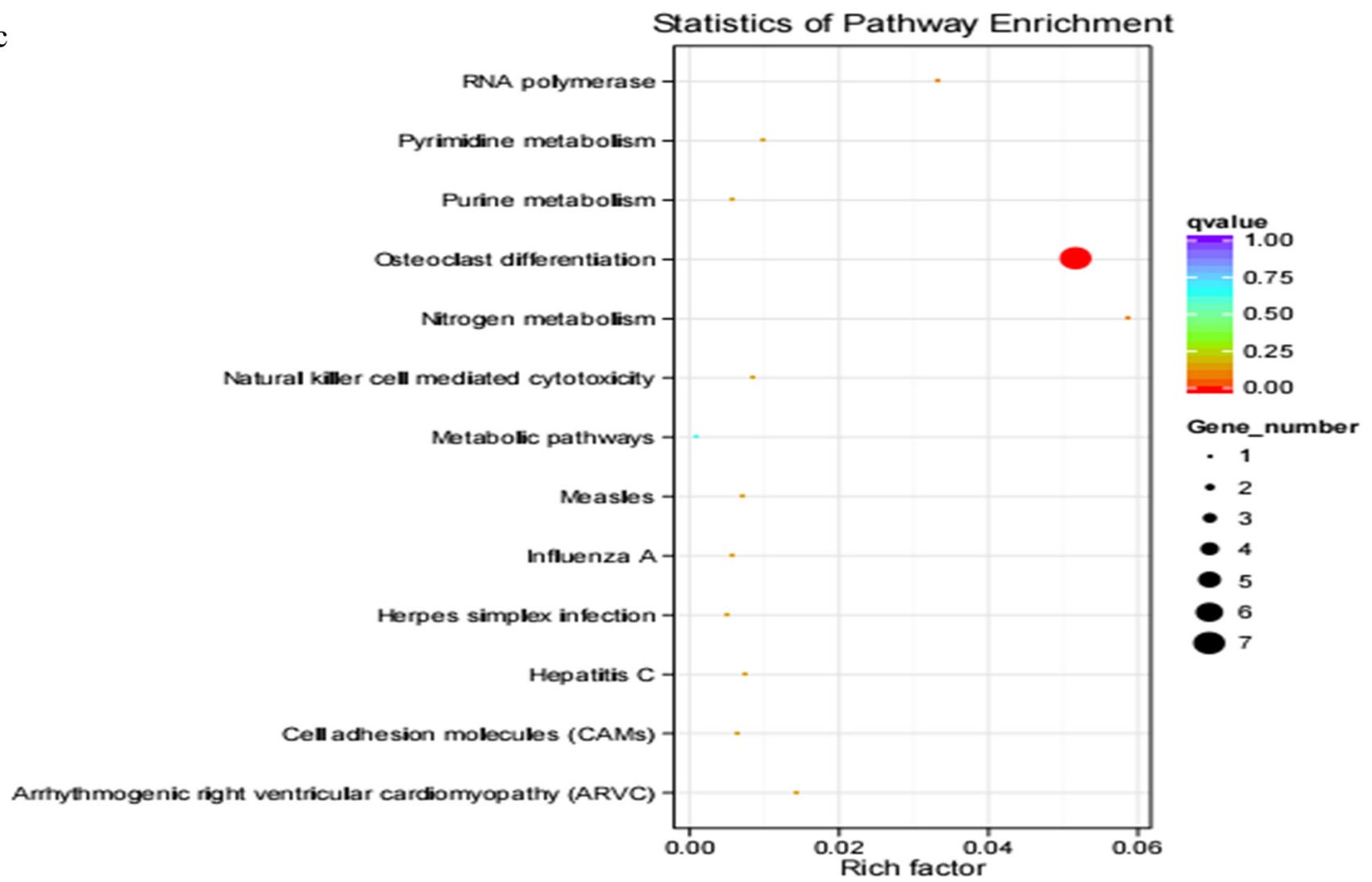

Figure 2. RNA sequencing results in pregnant and miscarriage cow groups at $18 \mathrm{~d}$ post-artificial insemination. Hierarchical clustering of differentially expressed genes (DEGs) showed that DEGs at $18 \mathrm{~d}$ were different between the pregnant cows and the cows who miscarried cows. (b) DEGs were screened in a volcano plot, and results suggested that 32 DEGs were found; 15 were upregulated and 17 were downregulated. (c) Kyoto encyclopedia of genes and genomes (KEGG) pathway analysis showed that DEGs were distributed in 13 pathways. 

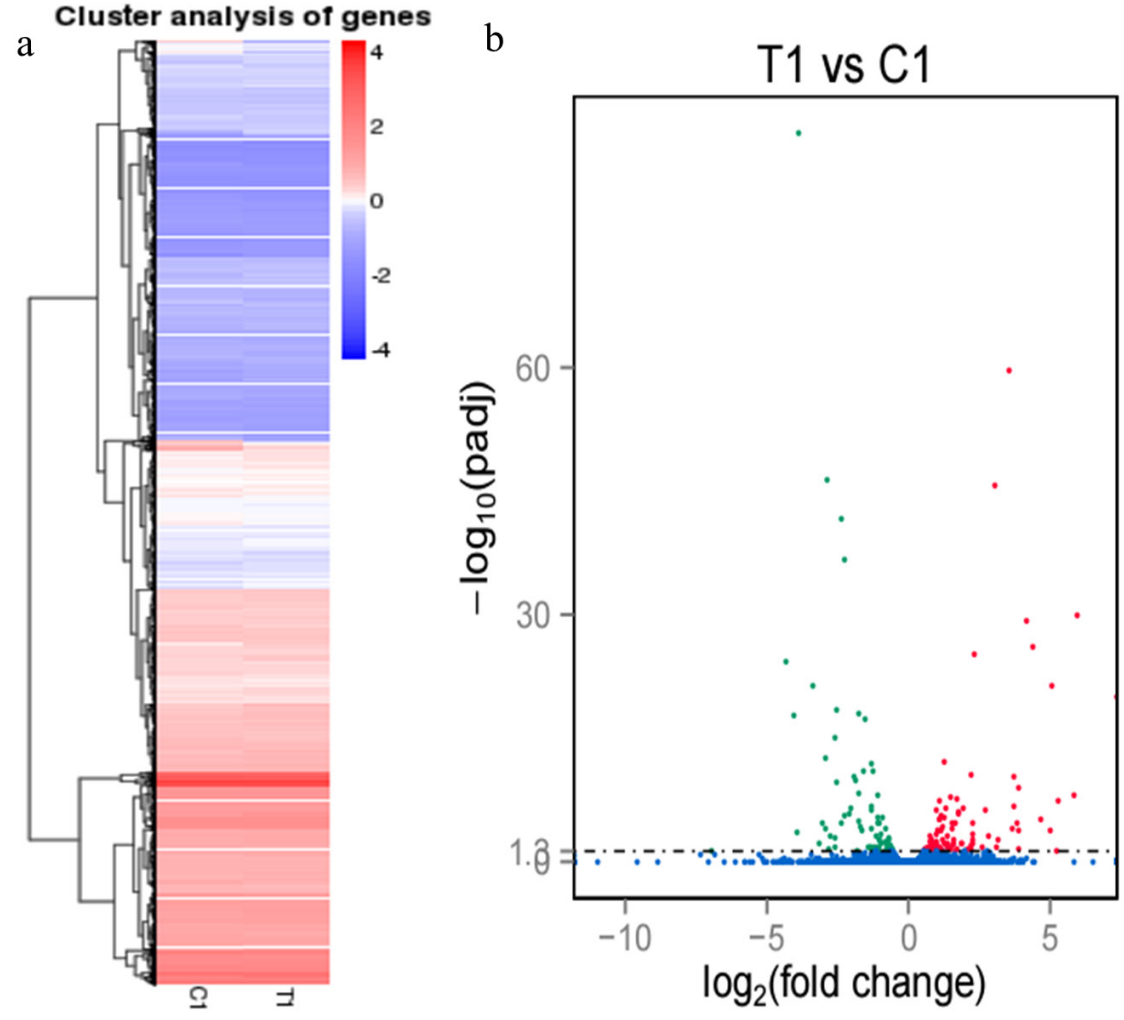

\section{Differential Expressed Genes ( 175 )}

- up regulated: 101

- down regulated: 74

c

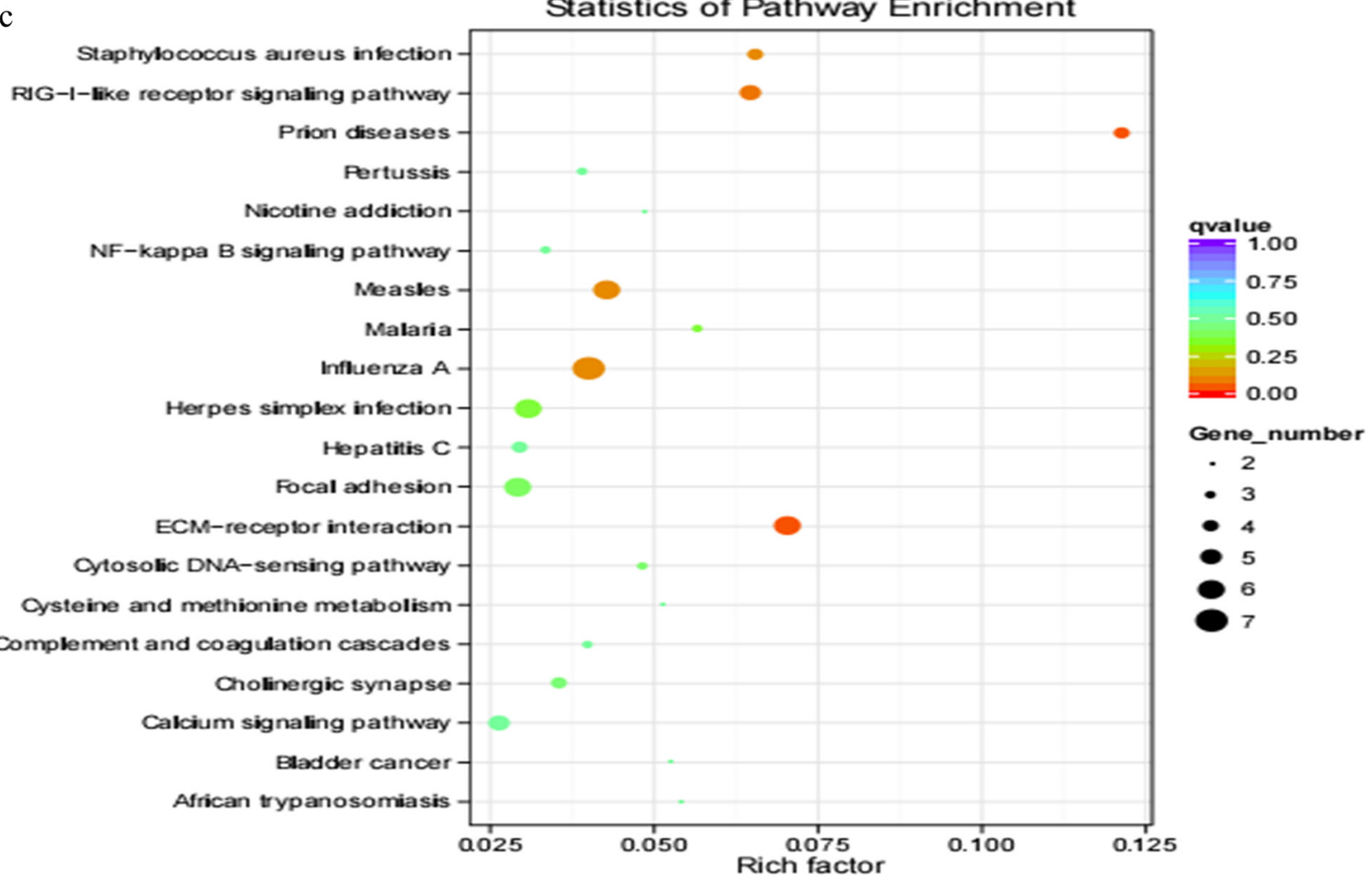

Figure 3. RNA sequencing results of pregnant cows and cows who miscarried at $21 \mathrm{~d}$ post-artificial insemination. (a) Hierarchical clustering of differentially expressed genes (DEGs) showed that DEGs at $21 \mathrm{~d}$ were different between the pregnant and miscarriage cows. (b) DEGs were screened in a volcano plot, and results suggested that 175 DEGs were found; 101 were upregulated and 74 were downregulated. (c) Kyoto encyclopedia of genes and genomes (KEGG) pathway analysis showed that DEGs were distributed in 20 pathways. 

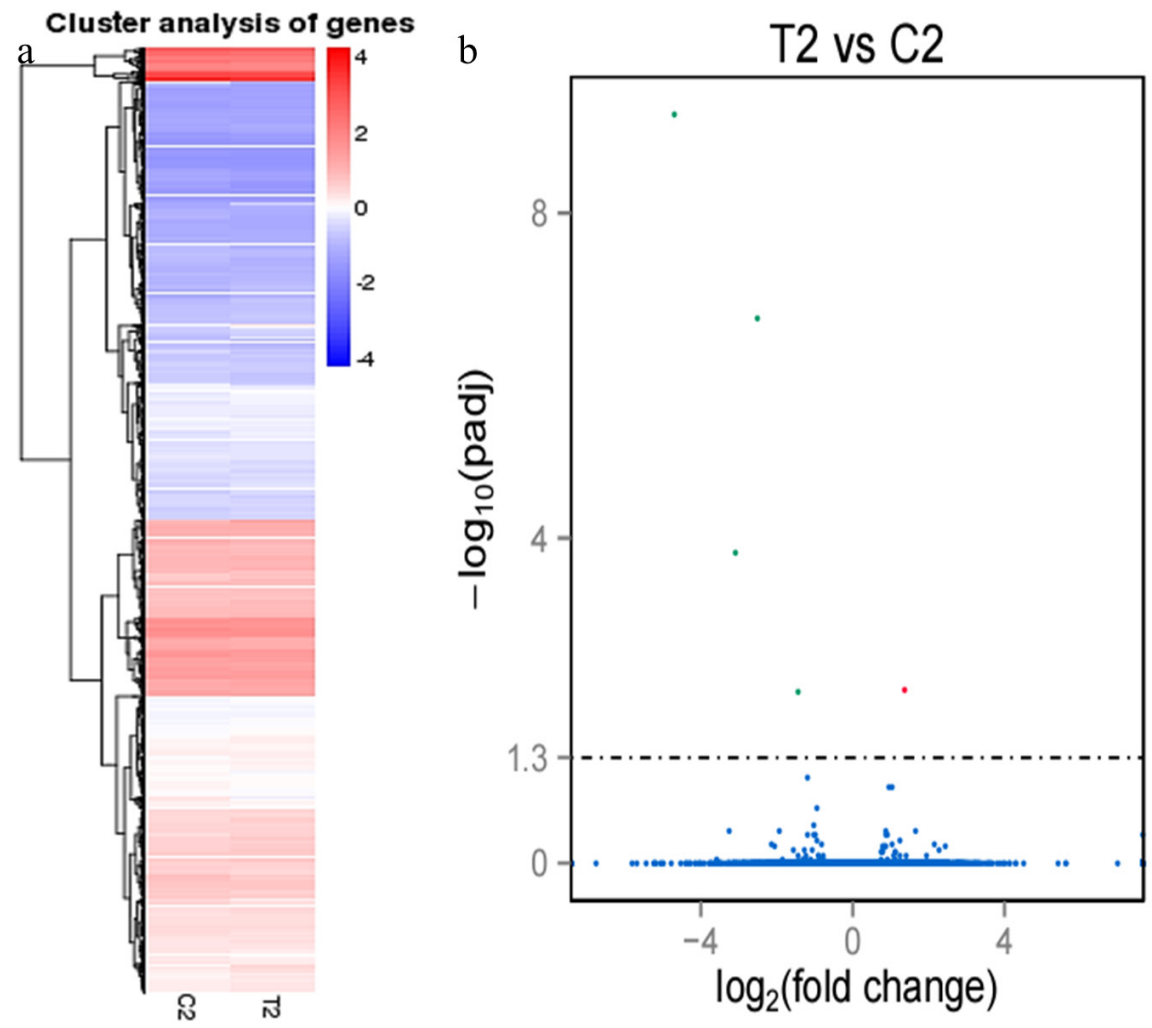

\section{Differential Expressed Genes ( 5 )}

- up regulated: 1

- down regulated: 4

c

Statistics of Pathway Enrichment

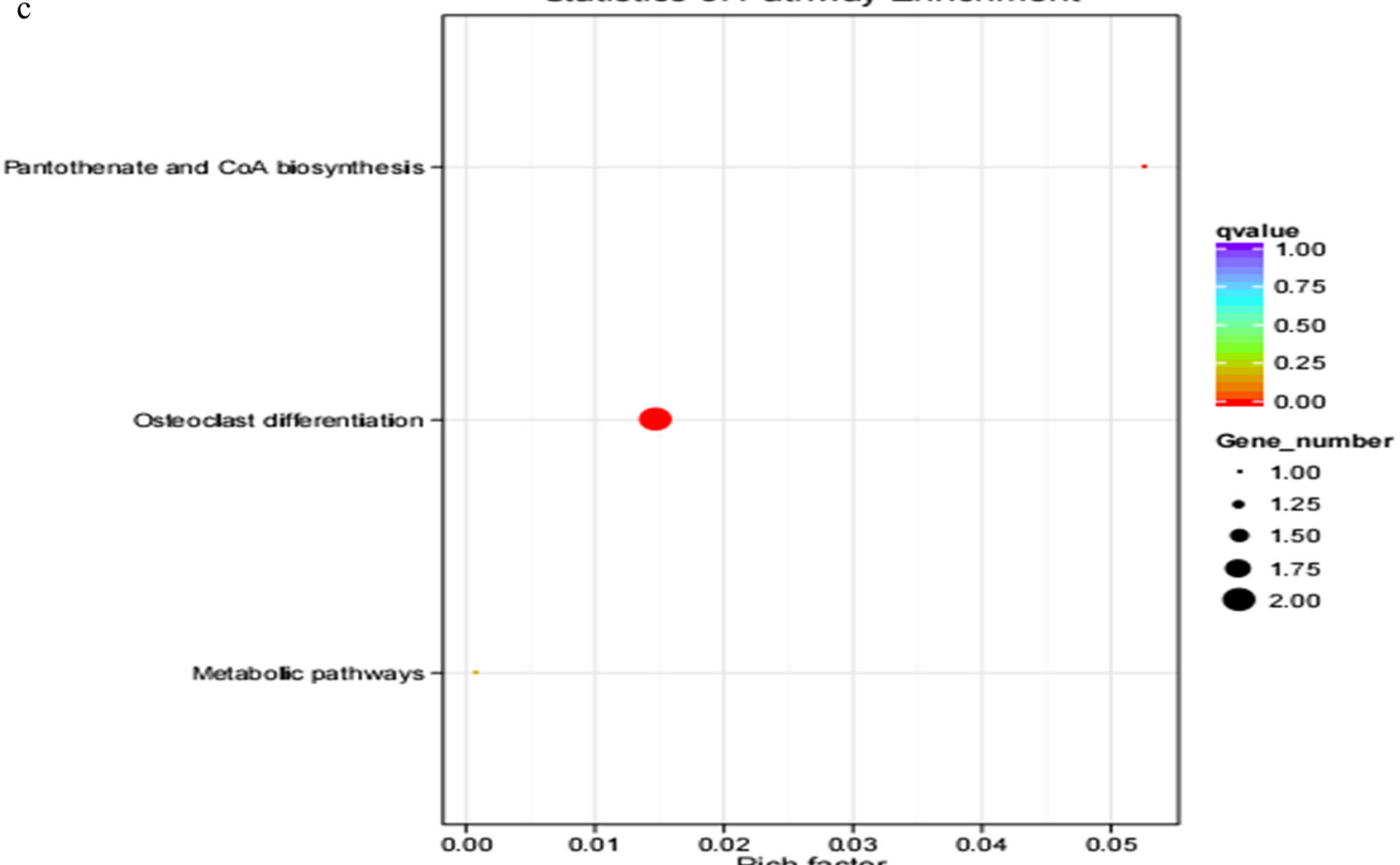

Figure 4. RNA sequencing results of pregnant cows and cows who miscarried at $33 \mathrm{~d}$ post-artificial insemination. (a) Hierarchical clustering of differentially expressed genes (DEGs) showed that DEGs at $33 \mathrm{~d}$ were weakly different between the pregnant cows and cows who miscarried. (b) DEGs were screened in a volcano plot, and results suggested that five DEGs were found; one was upregulated and four were downregulated. (c) Kyoto encyclopedia of genes and genomes (KEGG) pathway analysis showed that DEGs were distributed in three pathways. 

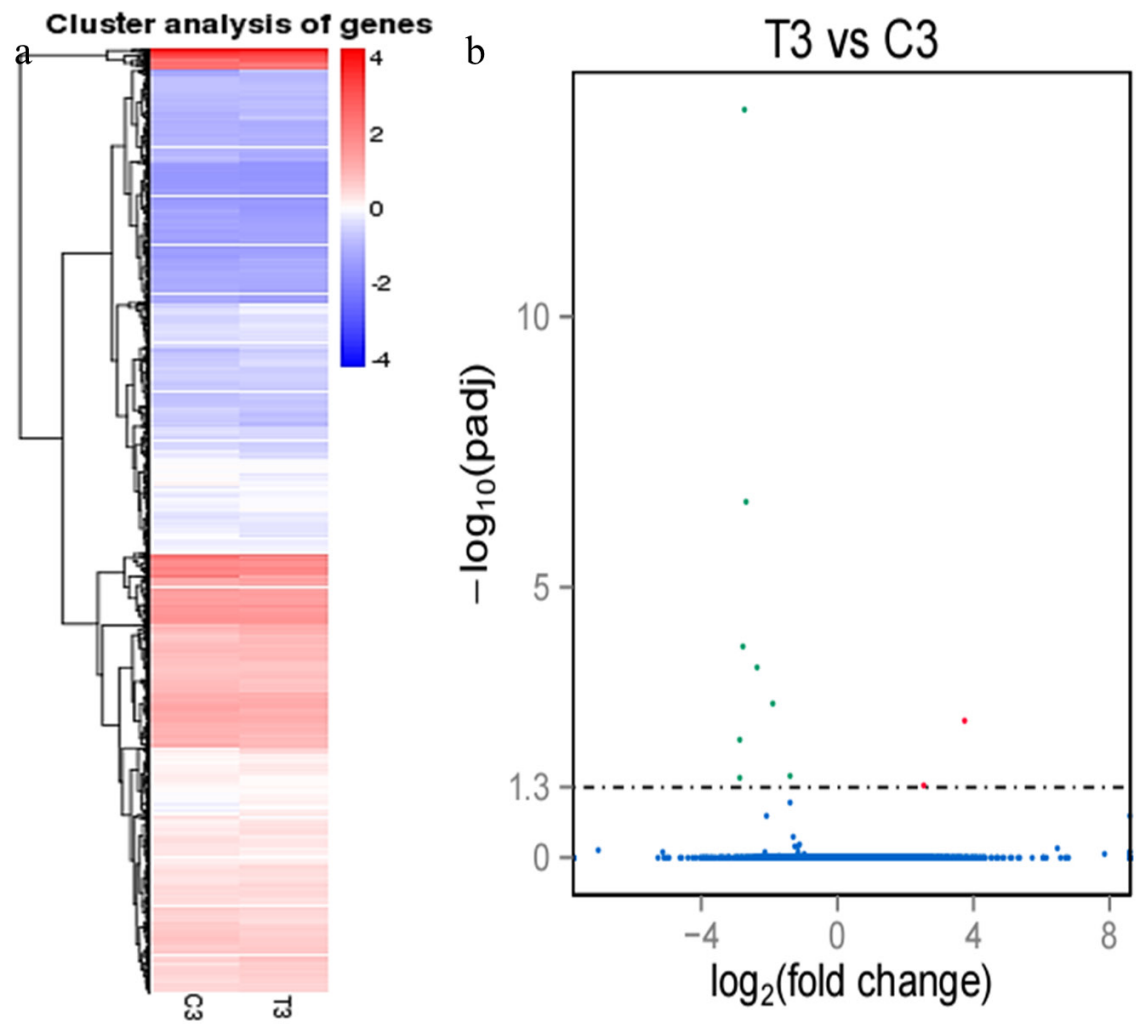

\section{Differential Expressed Genes ( 10 )}

- up regulated: 2

- down regulated: 8

C

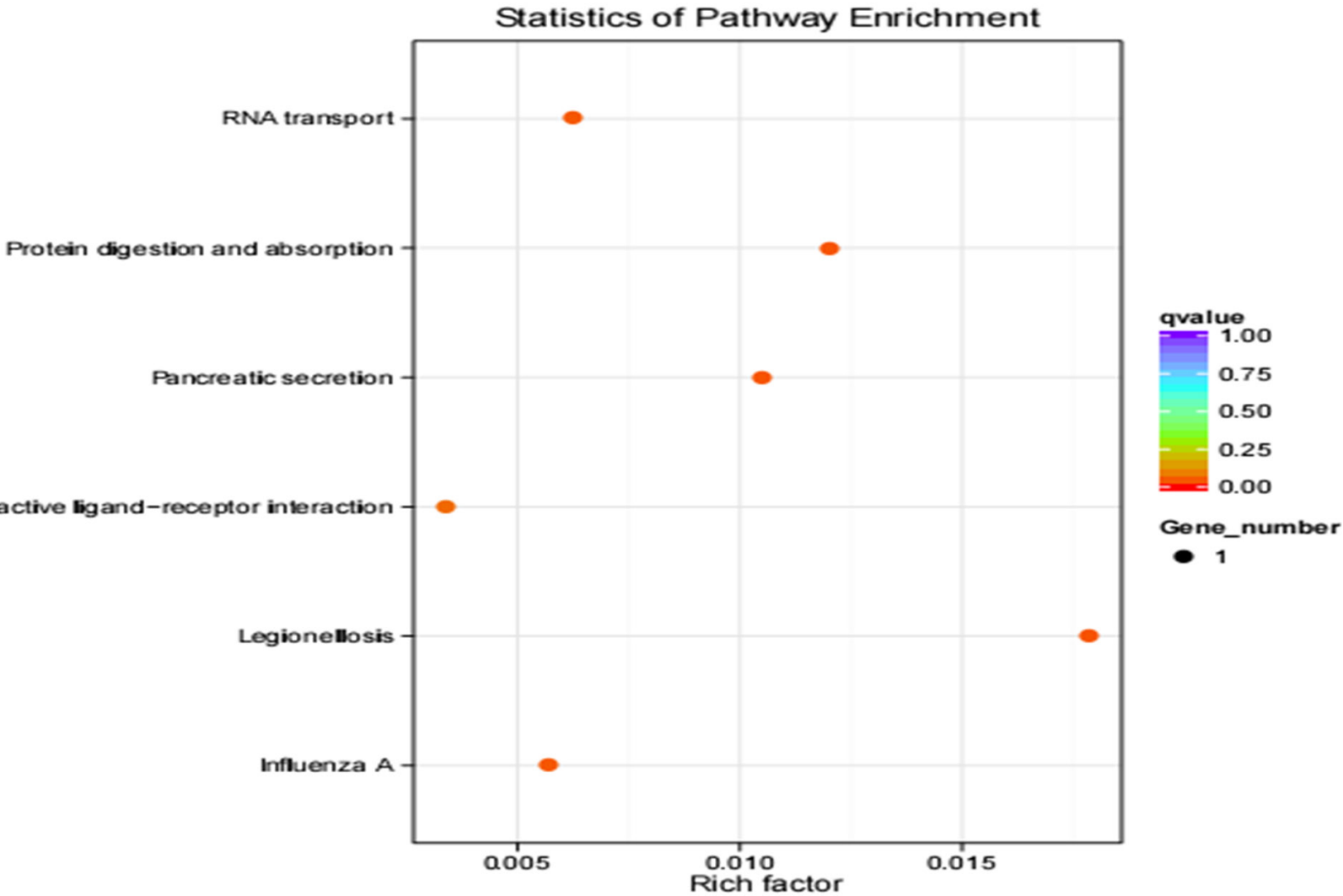

Figure 5. RNA sequencing results of pregnant cows and cows who miscarried at $39 \mathrm{~d}$ post-artificial insemination. (a) Hierarchical clustering of differentially expressed genes (DEGs) showed that DEGs at $39 \mathrm{~d}$ were weakly different between the pregnant cows and the cows who miscarried. (b) DEGs were screened in a volcano plot, and results suggested that 10 DEGs were found; 2 were upregulated and 8 were downregulated. (c) Kyoto Encyclopedia of Genes and Genomes (KEGG) pathway analysis showed that DEGs were distributed in six pathways. 
Cluster analysis of genes

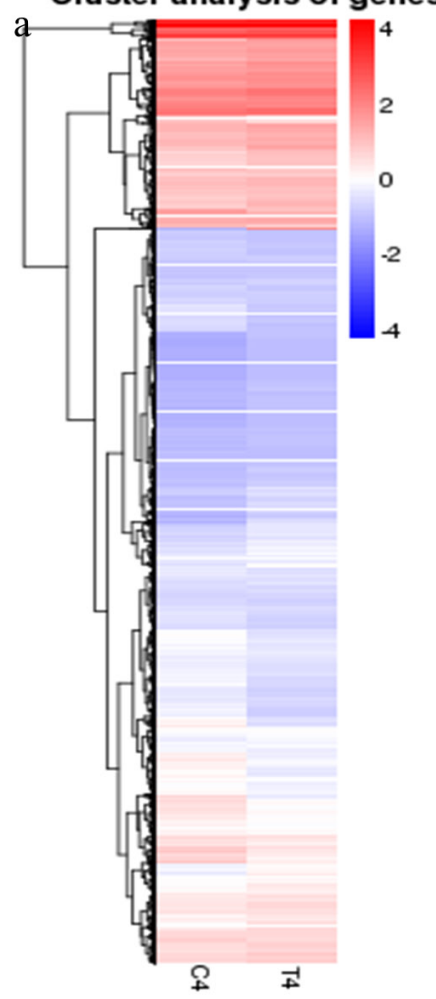

b

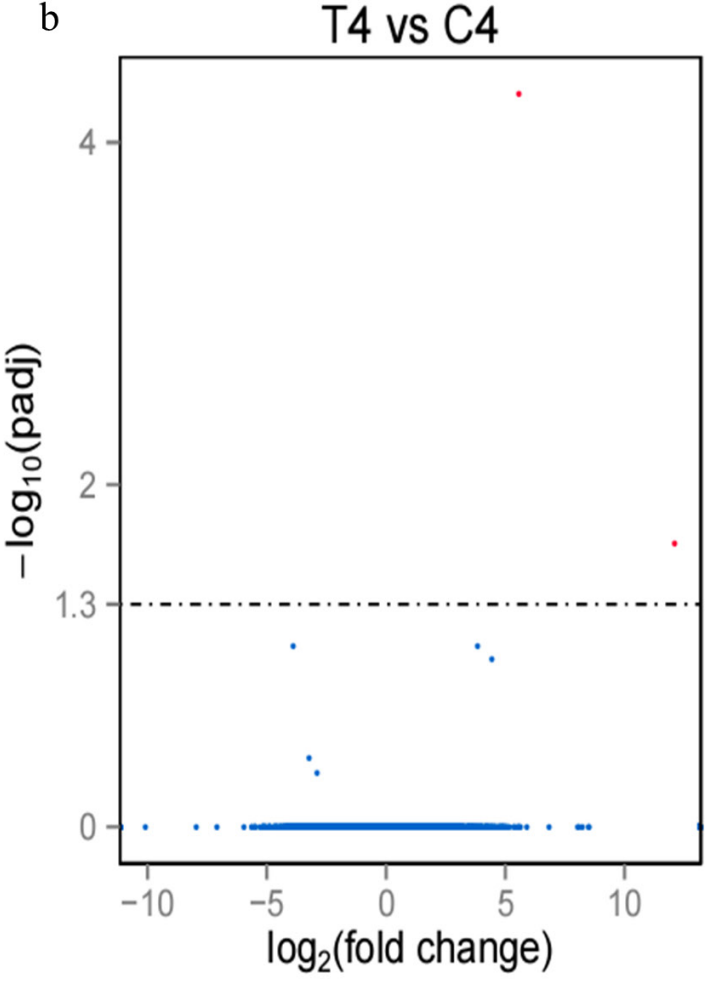

Differential Expressed Genes ( 2 )

- up regulated: 2

c

\section{Statistics of Pathway Enrichment}

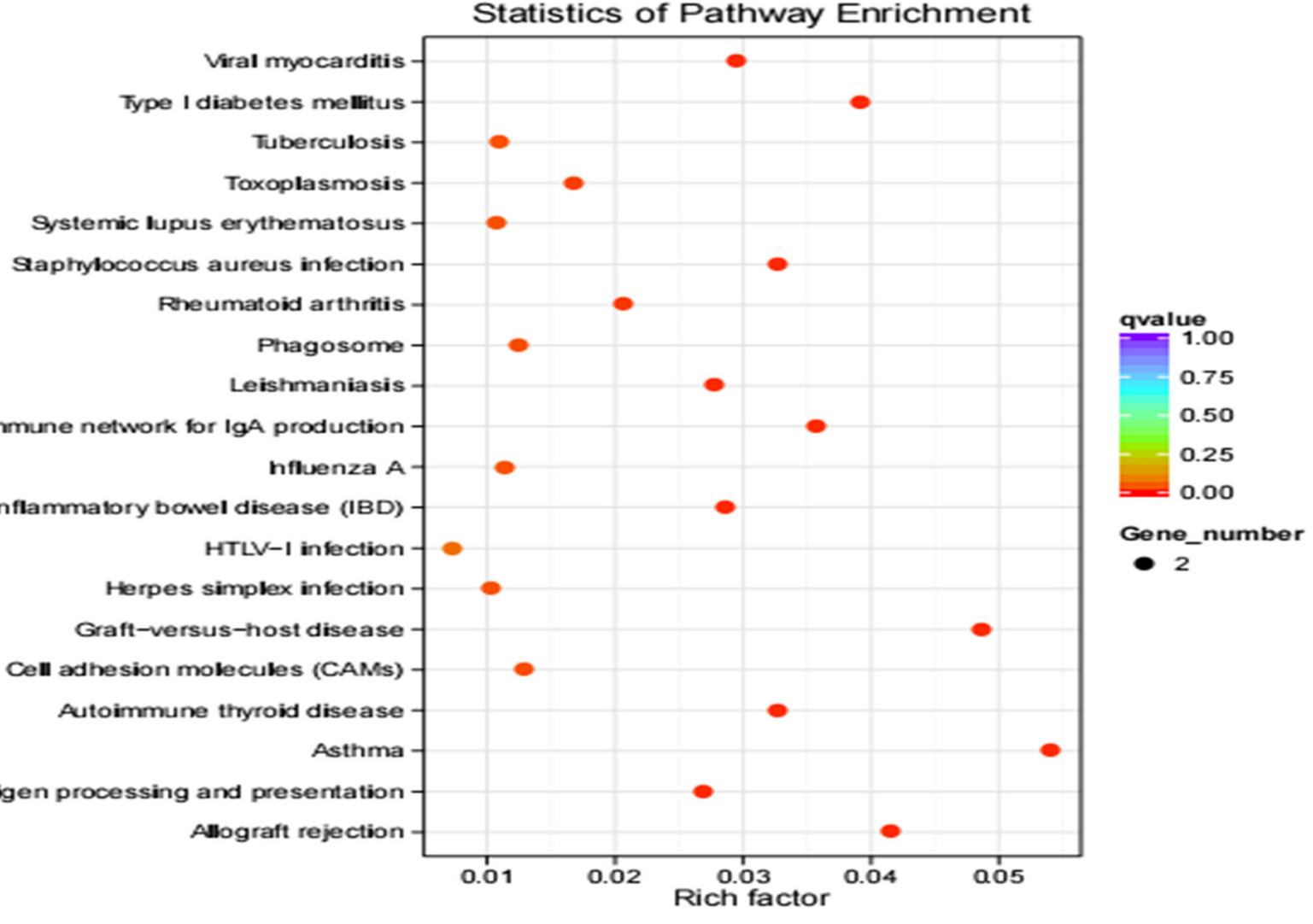

Figure 6. RNA sequencing results of pregnant and miscarriage cows at $51 \mathrm{~d}$ post-artificial insemination Hierarchical clustering of differentially expressed genes (DEGs) showed that DEGs at $51 \mathrm{~d}$ were weakly different between the pregnant cows and the cows who miscarried. (b) DEGs were screened in a volcano plot, and results suggested that two DEGs were found; two were upregulated and none were downregulated. (c) Kyoto Encyclopedia of Genes and Genomes (KEGG) pathway analysis showed that DEGs were distributed in 20 pathways. 


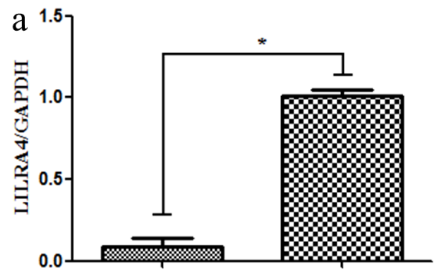

c
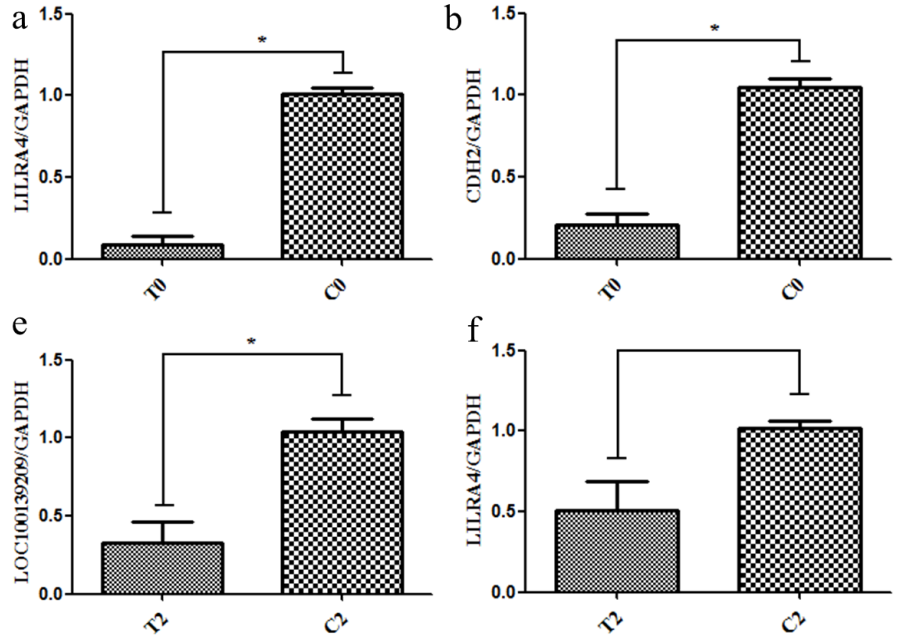

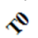

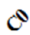

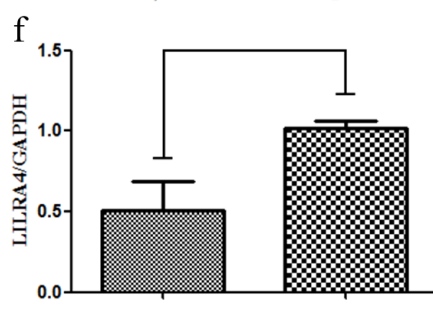

$s^{2}$

$c^{2}$

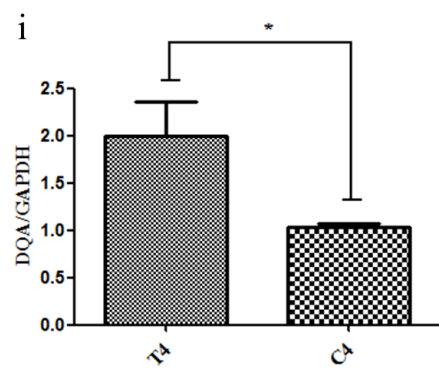

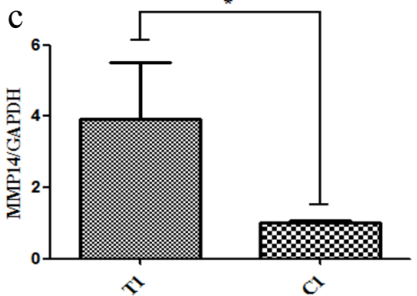
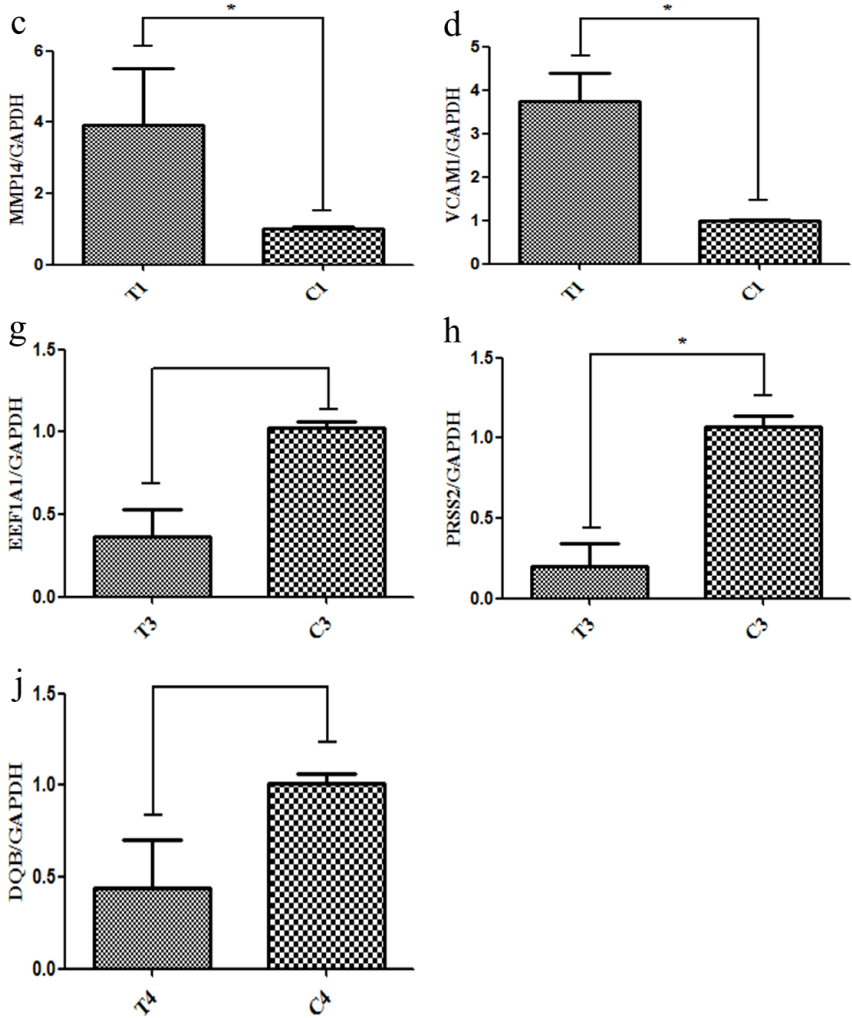

Figure 7. The differentially expressed genes (DEGs) were confirmed by real-time polymerase chain reaction (PCR). Two DEGs of LILRA4 (a) and CDH2 (b) at $18 \mathrm{~d}$, MMP14 (c) and VCAM1 (d) at $21 \mathrm{~d}$, LOC100139209 (e) and LILRA4 (f) at $33 \mathrm{~d}$, EEF1A1 (g) and PRSS2 (h) at $39 \mathrm{~d}$, and BoLA-DQA (i) and BoLA-DQB (j) at $51 \mathrm{~d}$ post-artificial insemination (Al) was further confirmed by real-time PCR. LILRA4, leukocyte immunoglobulin like receptor A4; CDH2, cadherin 2; MMP14, matrix metallopeptidase 14; VCAM1, vascular cell adhesion molecule 1; LOC100139209, leukocyte immunoglobulin-like receptor subfamily A member 6; EEF1A1, eukaryotic translation elongation factor 1 alpha 1; PRSS2, serine protease 2; BOLA-DQA, Bos taurus MHC class II antigen A. ${ }^{*} p<0.05,{ }^{* *} p<0.01,{ }^{* * *} p<0.001$.

DEGs in PI3K-Akt signalling pathway at $21 \mathrm{~d}$, and the expression profiles of COL6A3 suggested that it play an important role in pregnancy, and miscarriage was initiated by its abnormal expression [25]. In particular, MMP14, VCAM1, and MMP9 of DEGs in tumour necrosis factor signalling pathway further emphasized these genes during pregnancy, and MMP14 is important for maintenance of pregnancy in cattle [26]; VCAM1 is involved in the bovine conceptus adhesion to the uterine endometrium [27]. In addition, the Hippo signalling pathway play an important role in the preimplantation mouse development [28], and miscarriage was caused by the dysfunction of the Hippo signalling pathway.

In conclusion, our results identified genes and pathways crucial to pregnancy and miscarriage in cows.

\section{CONFLICT OF INTEREST}

We certify that there is no conflict of interest with any financial organization regarding the material discussed in the manuscript. Li Y, Huang L are employees of Helan Mountain Diary Company of Ningxia.

\section{ACKNOWLEDGMENTS}

This work was supported by China Agriculture Research System (CARS-36), the National Nature Science Foundation of China (31660645; 31760647); High level program for publishing paper of Ningxia University.

\section{REFERENCES}

1. Bai R, Kusama K, Sakurai T, et al. The role of endometrial selectins and their ligands on bovine conceptus attachment to the uterine epithelium during peri-implantation period. Biol Reprod 2015;93:46.

2. Dunne LD, Diskin MG, Sreenan JM. Embryo and foetal loss in beef heifers between day 14 of gestation and full term. Anim Reprod Sci 2000;58:39-44.

3. Berg DK, van Leeuwen J, Beaumont S, Berg M, Pfeffer PL. Embryo loss in cattle between Days 7 and 16 of pregnancy. Theriogenology 2010;73:250-60.

4. Diskin MG, Parr MH, Morris DG. Embryo death in cattle: an update. Reprod Fertil Dev 2011;24:244-51.

5. Carracedo S, Sacher F, Brandes G, Braun U, Leitges M. Redundant role of protein kinase $\mathrm{C}$ delta and epsilon during mouse 
embryonic development. PLoS One 2014;9:e103686.

6. Mitko K, Ulbrich SE, Wenigerkind H, et al. Dynamic changes in messenger RNA profiles of bovine endometrium during the oestrous cycle. Reproduction 2008;135:225-40.

7. Bai R, Bai H, Kuse M, et al. Involvement of VCAM1 in the bovine conceptus adhesion to the uterine endometrium. Reproduction 2014;148:119-27.

8. Li Q, Davila J, Bagchi MK, Bagchi IC. Chronic exposure to bisphenol a impairs progesterone receptor-mediated signaling in the uterus during early pregnancy. Receptors Clin Investig 2016;3:e1369.

9. Sasaki H. Roles and regulations of Hippo signaling during preimplantation mouse development. Dev Growth Differ 2017;59:12-20.

10. Newcomer BW, Cofield LG, Walz PH, Givens MD. Prevention of abortion in cattle following vaccination against bovine herpesvirus 1: A meta-analysis. Prev Vet Med 2017;138:1-8.

11. Terefe Y, Girma S, Mekonnen N, Asrade B. Brucellosis and associated risk factors in dairy cattle of eastern Ethiopia. Trop Anim Health Prod 2017;49:599-606.

12. Garrett JE, Geisert RD, Zavy MT, Morgan GL. Evidence for maternal regulation of early conceptus growth and development in beef cattle. J Reprod Fertil 1988;84:437-46.

13. Carter F, Forde N, Duffy P, et al. Effect of increasing progesterone concentration from day 3 of pregnancy on subsequent embryo survival and development in beef heifers. Reprod Fertil Dev 2008;20:368-75.

14. Clemente M, de La Fuente J, Fair T, et al. Progesterone and conceptus elongation in cattle: a direct effect on the embryo or an indirect effect via the endometrium? Reproduction 2009; 138:507-17.

15. Bauersachs S, Ulbrich SE, Gross K, et al. Embryo-induced transcriptome changes in bovine endometrium reveal speciesspecific and common molecular markers of uterine receptivity. Reproduction 2006;132:319-31.

16. Rhinehart JD, Starbuck-Clemmer MJ, Flores JA, et al. Low peripheral progesterone and late embryonic/early fetal loss in suckled beef and lactatingdairy cows. Theriogenology 2009; 71:480-90.
17. Rao X, Huang X, Zhou Z, Lin X. An improvement of the $2\left(^{-\Delta \Delta C T}\right)$ method for quantitative real-time polymerase chain reaction data analysis. Biostat Bioinforma Biomath 2013;3: 71-85.

18. Wolf E, Arnold GJ, Bauersachs S, et al. Embryo-maternal communication in bovine - strategies for deciphering a complex cross-talk. Reprod Domest Anim 2003;38:276-89.

19. Forde N, Carter F, Fair T, et al. Progesterone-regulated changes in endometrial gene expression contribute to advanced conceptus development in cattle. Biol Reprod 2009;81:784-94.

20. Carter F, Rings F, Mamo S, et al. Effect of elevated circulating progesterone concentration on bovine blastocyst development and global transcriptome following endoscopic transfer of in vitro produced embryos to the bovine oviduct. Biol Reprod 2010;83:707-19.

21. Clemente M, Lopez-Vidriero I, O'Gaora P, et al. Transcriptome changes at the initiation of elongation in the bovine conceptus. Biol Reprod 2011;85:285-95.

22. Imakawa K, Bai R, Fujiwara H, et al. Continuous model of conceptus implantation to the maternal endometrium. J Endocrinol 2017;233:R53-R65.

23. Psychoyos A. Hormonal control of ovoimplantation. Vitam Horm 1973;31:201-56.

24. Yamakoshi S, Bai R, Chaen T, et al. Expression of mesenchymal-related genes by the bovine trophectoderm following conceptus attachment to the endometrial epithelium. Reproduction 2012;143:377-87.

25. Diao H, Aplin JD, Xiao S, et al. Altered spatiotemporal expression of collagen types I, III, IV, and VI in Lpar3-deficient periimplantation mouse uterus. Biol Reprod 2011;84:255-65.

26. Sponchiado M, Gomes NS, Fontes PK, et al. Pre-hatching embryo-dependent and -independent programming of endometrial function in cattle. PLoS One 2017;12:e0175954.

27. Bai $\mathrm{R}, \mathrm{Bai} \mathrm{H}$, Kuse M, et al. Involvement of VCAM1 in the bovine conceptus adhesion to the uterine endometrium. Reproduction 2014;148:119-27.

28. Sasaki H. Roles and regulations of Hippo signaling during preimplantation mouse development. Dev Growth Differ 2017; 59:12-20. 